\title{
Precolouring in Compressive Spectrum Estimation for Cognitive Radio
}

\author{
Dimitrios Karampoulas ${ }^{1}$, Laurence S. Dooley ${ }^{2}$, Soraya Kouadri Mostéfaoui ${ }^{3}$ \\ Next Generation Multimedia Technology Group (XGMT), Department of Communication and Systems, \\ The Open University, Milton Keynes, United Kingdom \\ ${ }^{1}$ dkarampoulas@gmail.com \\ 2 laurence.dooley@open.ac.uk \\ 3 s.kouadri@open.ac.uk
}

\begin{abstract}
One of the major challenges in cognitive radio (CR) networks is the need to sample signals as efficiently as possible without incurring the loss of vital information. Compressive Sensing (CS) is a new sampling paradigm which provides a theoretical framework for sub-sampling signals which are characterized as being sparse in the frequency domain. The random demodulator (RD) is a CS-based architecture which has been employed to acquire frequency sparse, bandlimited signals which typify the signals which often occur in many CR-related applications. This paper investigates the impact of precolouring upon CS performance by combining the RD with an autoregressive (AR) filter model to enhance compressive spectral estimation. Quantitative results with quadrature phased shift keying (QPSK) modulated multiband signals, corroborate that adopting a precolouring strategy both reduces the spectral leakage in the power spectrum, and concomitantly improves the overall signal-to-noise ratio (SNR) performance of the compressive spectrum estimator.
\end{abstract}

Key words: Cognitive Radio, Compressive Sensing, Random Demodulator, Autoregressive Model, Precolouring, Signal to Noise Ratio

\section{INTRODUCTION}

Cognitive radio (CR) is broadly defined as a netwotk which is able to sense its spectral environment over a wide frequency band and opportunistically provide wireless links to best meet the users' requirements, without causing harmful interference [1] and [2]. A key CR design objective is the development of suitable spectral detection and estimation techniques able to efficiently sense and identify the available spectrum. One of the major challenges of conventional spectrum estimation methods [3] is the high sampling rates involved. This has given impetus to the emergence of compressive sensing (CS) techniques, which are a new sampling paradigm $[4,5,6]$ for data acquisition. Furthermore, the stringent timing requirements for rapidly sensing dynamically changing spectra, means only a small number of measurements are acquired from the received signal, which may be insufficient for reliable signal reconstruction.

As delineated in both [1] and [7], only a small portion of bands in the radio frequency (RF) spectrum are heavily used, while others are only either partially or rarely occupied. As a consequence, in open-spectrum networks, the wireless signals are typically sparse in the frequency domain, that is, the number of significant frequency components is often much smaller than the band limit allows. More specifically, in many RF applications, signals with a large bandwidth may only have a small 'information rate' [8]. (CS) affords an effective means for recovering sparse signals, by requiring the availability of only a relatively small number of non-adaptive measurements to reconstruct the signals which are compressible by for instance, the Fourier or Wavelet transforms. The randomizing of these measurements plays an integral role in underpinning CS theory, since it leads to very effective sensing mechanisms, as will be explained further in Section II-A.

One particular CS technique is the analog-to-information converter (AIC), realization known as the random demodulator (RD) [9-11]. The $\mathrm{RD}$ structure is able to efficiently recover discrete, multitone signals which are characterized by being bandlimited, periodic and sparse. In [12], it is claimed that by windowing the input signal to the $\mathrm{RD}$, broader signal classes, such as non-harmonic tones and multiband signals can be approximated by periodic multitone signals, though for multiband signals, which are typical of the types of signals which occur in many CR applications, the number of frequencies required for the approximation is linearly dependent on the bandwidth. It is important there are sufficient numbers of frequencies within each band to avoid recovery errors, though this has a commensurate impact upon the computational load of both the sampling and recovery stages [13]. One other limitation of the RD structure highlighted in [13] is the maximal frequency that is able to be recovered is only around $500 \mathrm{kHz}$.

CR inherently seeks to exploit unoccupied portions of the spectrum [2] and since multiband signals can be approximated by periodic multitone signals, the RD can be considered as undertaking spectrum sensing in certain scenarios [12]. The $\mathrm{RD}$ has previously been used for the spectrum sensing of sparse bandlimited signals $[14,15,16]$, with spectrum estimation accomplished by identifying the sub-carriers of the occupied bands. It has not been applied to the recovery of the power spectrum density (PSD) of a signal and this provided 
the motivation to investigate modifying the basic $\mathrm{RD}$ architecture to achieve this objective for CR-related signals.

This paper presents a novel CS-based technique which employs the RD to recover the PSD of frequency sparse multiband signals which are characterised by having either one or more continually occupied bands, while concomitantly occupying a relatively small portion of the available spectrum. The input signal is firstly precoloured using an autoregressive (AR) model instead of windowing, with the RD then applied, to increase the spectral dynamic range and emphasize strong frequency components. Precolouring has the effect of enhancing signal sparsity by sharpening dominant frequencies while attenuating those weaker spectral components which lie outside the bands of interest. This augments signal sparsity in the frequency domain and facilitates better PSD recovery. It needs to be stressed however, that the design aim is not to exactly recover the signal PSD, but to efficiently identify occupied spectral bands, which is the key objective in CR systems.

To evaluate this CS technique, quadrature phased shift keying (QPSK) test signals were analysed as these are proposed in the IEEE 802.22 standards for CR networks [17], [18].The results testify the effectiveness of applying precolouring to improve the performance of the proposed compressive spectrum estimator, both in terms of spectral leakage and signal to noise ratio (SNR). Furthermore, precolouring enhances the robustness of the proposed spectrum estimator to both input noise and in being able to successfully identify occupied spectral bands when the signal is under-sampled as low as $5 \%$ of the Nyquist rate.

The remainder of the paper is organized as follows. Section II presents a brief overview of both CS theory and the basic RD architecture, while Section III describes the concept of precolouring and the AR model. A results analysis is presented in Section IV, with some concluding comments being provided in Section V.

\section{COMPRESSIVE SENSING AND THE RANDOM DEMODULATOR}

\section{A. Compressive Sensing Background}

CS is a signal processing technique which acquires and accurately reconstructs signals without upholding the basic tenet of the Nyquist sampling theorem [4]. It solves an underdetermined set of linear equations, with more unknowns than equations for which an infinite number of possible solutions exist. To resolve this problem, additional constraints are imposed upon the system, with one such constraint being sparsity in a particular domain. A signal is defined as sparse, if it contains many coefficients either close or equal to zero when represented in some domain [4], such as time or frequency. Without loss of generality, in this paper it is assumed the domain in which sparsity occurs is the frequency domain or basis and the corresponding basis matrix is the normalised discrete Fourier transform (DFT) matrix.

Compressed sensing takes a small set of linear, nonadaptive measurements using a matrix basis $\boldsymbol{\Phi}$ which is largely incoherent with the basis in which the signal is known to be sparse, which is assumed to be $\boldsymbol{\Psi}$. Incoherence between $\boldsymbol{\Phi}$ and $\boldsymbol{\Psi}$ means that if the signal of interest is sparsely represented in the $\boldsymbol{\Psi}$ domain, then it has a dense representation in the $\boldsymbol{\Phi}$ domain [4] and vice versa.

Hence, if a discrete-time signal vector $x$ of length $N$ that is $K$-sparse in $\boldsymbol{\Psi}$ with dimensions $N \mathrm{x} N$, then for the signal to be represented in the $\boldsymbol{\Psi}$ domain by the vector $\alpha$, the following must hold:

$$
x=\Psi \alpha
$$

Since the measurements are taken on a basis $\boldsymbol{\Phi}$ which is incoherent with $\boldsymbol{\Psi}$, if the measurements are represented by a vector $y$ of length $M<<N$, then the following is valid:

$$
y=\boldsymbol{\Phi} x
$$

Combining (1) and (2) gives:

$$
y=\boldsymbol{\Phi} x=\boldsymbol{\Phi} \Psi \alpha=\mathbf{V} \alpha
$$

where $\mathbf{V}=\boldsymbol{\Phi} \boldsymbol{\Psi}$ an $M \mathrm{x} N$ matrix.

As (3) is an underdetermined linear system of equations, there are an infinite number of possible vectors $\alpha$. CS theory states that due to the signal sparsity in the $\boldsymbol{\Psi}$ domain and the incoherence between bases $\boldsymbol{\Phi}$ and $\boldsymbol{\Psi}$, (3) can be solved and $\alpha$ recovered by $l_{1}$-norm minimization with high probability[4], [7], [8]:

$$
\min \|\alpha\|_{1} \quad \text { s.t. } \quad y=\boldsymbol{\Phi} \boldsymbol{\Psi} \alpha=\mathbf{V} \alpha
$$

This equation can be solved using linear programming techniques [19].

Any random matrix having a Gaussian, Bernoulli, Rademacher or any other sub-Gaussian distribution, will by definition be incoherent with any fixed basis $\boldsymbol{\Psi}$ [4], [20]. It is this fundamental precept which is exploited by the RD, as it uses a random matrix to sense the input signal by acquiring measurements $y=\boldsymbol{\Phi} x$. Reconstruction is achieved by solving (4) where as alluded, above the fixed basis $\boldsymbol{\Psi}$ is the DFT matrix. The RD architecture will be described in the next section.

\section{B. The Random Demodulator}

The basic RD structure is shown in Figure 1 and has three constituent components: demodulation, filtering and uniform sampling [12], [21]. The input signal is modulated by a square pulse train of random values $\{ \pm 1\}$ generated by a pseudorandom number (PN) sequence. This is called the chipping sequence $p_{c}(t)$ which must alternate between values at least as fast as the Nyquist frequency of the input signal. The demodulation smears the energy of the signal tones across the entire spectrum, giving each a unique spectral signature that can be discerned by the second stage which is a low-pass filter implemented as an integrator. The final stage is an 
analogue-to-digital converter (ADC) which is clocked at a sampling rate of $M<N$. As the RD directly acquires compressive measurements without firstly having to sample the continuous signal $x(t)$, this is equivalent to a system which samples $x(t)$ at

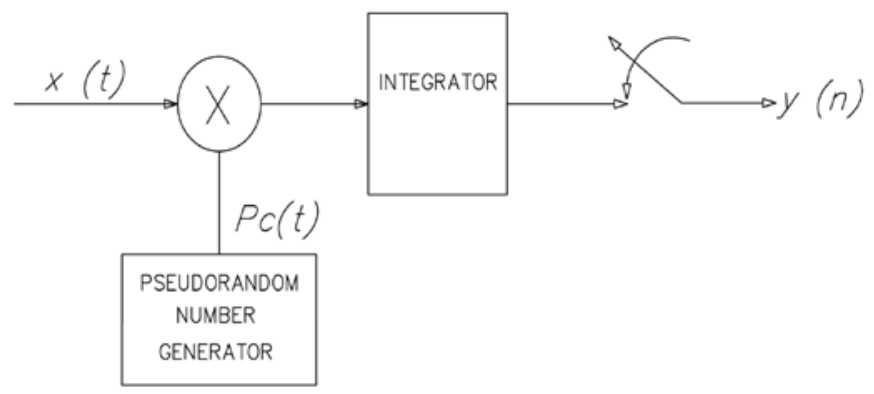

Figure 1. Random Demodulator Block Diagram

the Nyquist rate to yield a discrete-time vector $x$, before then applying $\boldsymbol{\Phi}$ to obtain the measurements $y=\boldsymbol{\Phi} x$ [21]. The measurement process is the dot product of $x$ with the random sequence $p_{c}(t)$ for each sequential block of $N / M$ coefficients. $\boldsymbol{\Phi}$ is a $M \times N$ matrix and containing $N / M$ randomly generated \pm 1 values per row, so for example, with $N=9$ and $M=3$, $\Phi$ will be:

$$
\boldsymbol{\Phi}=\left[\begin{array}{ccccccccc}
1 & -1 & 1 & 0 & 0 & 0 & 0 & 0 & 0 \\
0 & 0 & 0 & 1 & 1 & -1 & 0 & 0 & 0 \\
0 & 0 & 0 & 0 & 0 & 0 & -1 & -1 & 1
\end{array}\right]
$$

The output $y(n)$ from the RD can be considered as a linear transformation of the discrete vector $\alpha$. This transformation is expressed as $\mathbf{V}=\boldsymbol{\Phi} \boldsymbol{\Psi}$ with the signals being sparse in the frequency domain.

\section{PRECOLOURING}

As highlighted in Section II-A, constraints must be assumed for the signals of interest for the underdetermined set of linear equations to be solved. In this paper, the key constraint enforced is frequency sparsity, though one of the key motivations for precolouring is to reduce both the leakage of dominant spectral components and increase the dynamic range of the spectrum. This has the benefit of making the signal spectrum sharper, so enhancing signal sparsity by eliminating the weaker spectral components which lie outside the bands of interest.

Signal colouring is accomplished using an AR model of order $p[22,23]$. It is important to stress the rationale for choosing an AR filter is not spectral estimation per se [22,23], but instead to partially colouring the input signal to reduce spectral leakage and increase the dynamic range, thereby performing better intermediate CS spectrum estimation.This paper, adopts the same idea as in [22,23], but applies AR filter for signal precolouring instead of prewhitening. The influence of the precolouring filter is subsequently removed by inverse filtering of the intermediate spectrum estimate.

The output of a $p^{\text {th }}$ order AR filter is given by:

$$
y(n)=x(n)+\sum_{k=1}^{p} a_{k} y(n-k)
$$

where $\alpha_{\mathrm{\kappa}}$ are the AR filter coefficients which are optimally estimated using the modified covariance method [24] and the intermediate spectrum $S_{y}(f)$ of $\mathrm{y}(n)$ is derived using established spectral estimation techniques [17], such as the classic Periodogram and Correlogram estimators. The RD is applied for the ensuing spectral estimation and signal recovery, before the spectrum $S_{x}(f)$ is estimated from $S_{y}(f)$ by inverse filtering as follows:

$$
S_{x}(f)=\frac{S_{y}(f)}{\left|1+\sum_{k=1}^{p} a_{k} e^{-j 2 \pi f k}\right|^{-2}}
$$

The reason for the lower spectral leakage when an AR filter is used is due to the properties of an AR spectral estimator [24]. The AR power spectrum $S_{x}(f)$ is the Fourier equivalent of the corresponding autocorrelation sequence. Since the AR order is $p$, for the Correlogram-based spectrum estimator, autocorrelation coefficients having lags greater than $p$ are zero. This is not the case for the AR spectrum estimator where a nonzero extrapolation generates the unavailable lags as there is no windowing of the autocorrelation sequence as with the classic spectral estimators. The corollary is AR-based PSD does not exhibit the sidelobe phenomena and the resultant deleterious effects of spectral leakage, as in the classical spectral estimators.

\section{SIMULATION RESULTS}

To evaluate the performance of the new RD-based CS system architecture shown in Figure 2, experiments were undertaken upon a MATLAB-based platform. Two QPSK modulated random binary sequences of duration $0.3 \mathrm{sec}$, having a bit-rate of 215bps together with two carrier frequencies at $1 \mathrm{kHz}$ and $2.5 \mathrm{kHz}$ respectively were used as the test inputs. These carrier frequencies take cognizance of the maximal frequency recovery capability of the RD model highlighted in Section I. The sampling frequency was chosen 1.67 times greater than the Nyquist rate which corresponds to a signal length of 2048 samples. The CS performance was then analysed at sub-sampled rates of $68 \%, 34 \%, 17 \%, 8.5 \%$ and $4.25 \%$ of the Nyquist rate which corresponds to 1024 , 512, 256, 128 and 64 samples respectively. As the input $x(n)$ is precoloured, the input to the RD model is the intermediate signal $y(n)$ in (5).

It is assumed additive white Gaussian (AWG) noise has been added to $x(n)$, so that input SNR was $8.1 \mathrm{db}$ as prescribed in the proposed IEEE 802.22 standards for CR [17],[18]. 


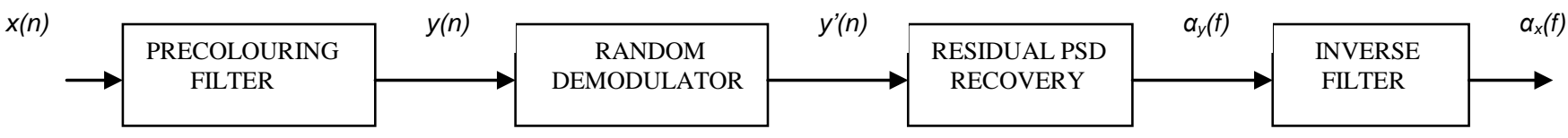

Figure 2. Compressive Sensing Block Diagram with Precolouring

A $4^{\text {th }}$ order AR model was empirically chosen for these experiments. This represented a design a compromise, since higher-order AR filters not only increase the processing overheads, but also the spectral leakage. Additionally, lower AR filter order meant that no stability problems related to the inverse filter could be encountered in the experiments.

It is important to emphasize that from a CR perspective, the aim is to recover the signal PSD in such a way that the occupied bands are identified, and not the reconstruction of the original signal.

The precolouring stage does not have to be integrated into the CS structure of Figure 2. It could equally for instance, be implemented by the primary user in a CR network. This would avoid the requirement for a high-speed analog to digital converter to be implemented within the AR filter structure, which would negate the benefit derived by using the lowersampling rate of the RD.

For the specific test signals there are two occupied bands having a bandwidth of $215 \mathrm{~Hz}$ at $1 \mathrm{kHz}$ and $2.5 \mathrm{kHz}$ respectively. To analyze the effect of precolouring in the proposed RD-based CS system, the signal energy in the bands of interest is measured at various sampling frequencies below the Nyquist rate. The corresponding results are shown in Figure 3, where the percentage of the total signal energy content within the bands of interest is termed the PSD spectral concentration. The corresponding spectral leakage for the bands of interest is then the difference from the ideal PSD spectral concentration (100\%).

The results reveal that when precolouring is applied across the range of sub-sampling rates, the average spectral concentration improvement achieved is approximately $48 \%$ better than when it is not applied, i.e. the case of the classic Periodogram solution. As the highlighted example in Figure 3 shows, the spectral leakage is lower than $\approx 10 \%$ of the total signal energy in the precolouring case, even when the sampling frequency falls to $20 \%$ of the original Nyquist rate compared with more than $35 \%$ of the signal energy when no precolouring is employed. Overall, the spectral leakage across the range of sub-sampled rates provides an average improvement in the PSD spectral concentration of $47.8 \%$ when the proposed CS-model is applied.

Figure 4 displays the corresponding SNR performance of the proposed system (Figure 2). The SNR is given by:

$$
S N R_{s}=10 * \log \frac{\|P\|_{2}}{\left\|P-a_{x}\right\|_{2}}
$$

where $P$ is the signal PSD when it is either sampled at or above the Nyquist rate, and $\alpha_{x}$ is the PSD of the undersampled CS signal (see Figure 2). The results confirm the superior SNR performance when precolouring is applied, with for instance a higher SNR of $12.3 \mathrm{db}$ at $70 \%$ of the Nyquist sampling rate and despite the more rapid roll-off, the system SNR is still $7.5 \mathrm{db}$ better at $40 \%$ of the Nyquist rate compared with when no precolouring is applied, to underscore the superior SNR behaviour of the system when precolouring is applied. Note, for the highly under-sampled scenario of $<5 \%$ of the Nyquist rate, the SNR graphs converge, though the sampling rate is now too low to pragmatically collect a sufficient number of samples.

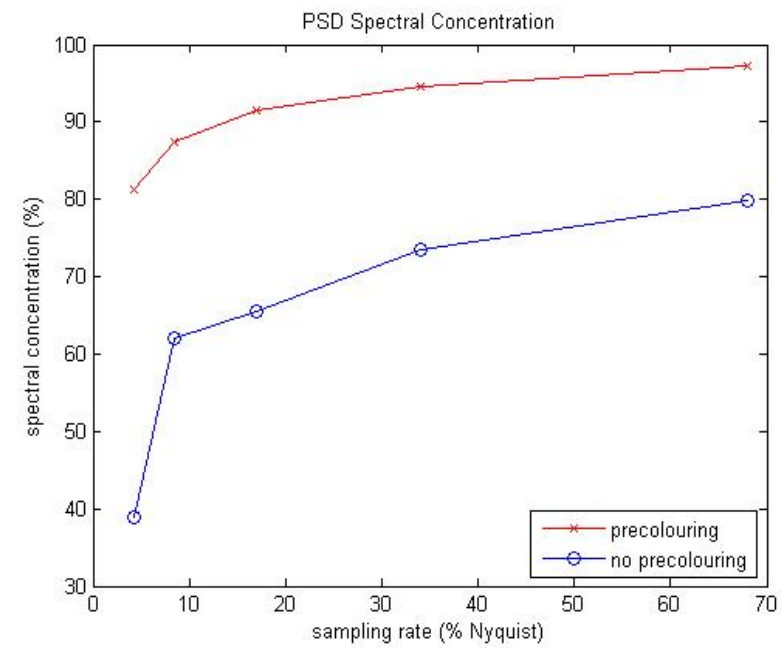

Figure 3. The effect of precolouring on the PSD spectral concentration

Figures 5 and 6 respectively plot the recovered PSD both with and without precolouring at sampling rates 68\% and $4.25 \%$ of the Nyquist rate. The spectral leakage is evidently less pronounced when precolouring is applied, particularly at the lower rate, where it is still possible for the occupied bands to be identified. In contrast, when precolouring is not applied, the spectral leakage is more pronounced and at the lower sampling rate the occupied bands are now unable to be identified. Finally, Figures 7(a) and 7(b) analyse the performance of the proposed RD-based CS structure at different AWG noise levels. The input SNR levels were defined as in the IEEE 802.22 standards for cognitive radio networks [17], [18]. Figure 7(a) reveals that the spectral leakage is always $<20 \%$ in the case of precolouring, whereas it remains steadily above $25 \%$ for the no precolouring case. Figure 7(b) also demonstrates that precolouring consistently provides improved SNR performance in the RD structure, with the system SNR when precolouring is applied being at least a factor of 2 higher compared with the no precolouring scenario. Moreover, it is evident from these results that system SNR increases significantly at lower AWG noise levels, in 
marked contrast to the non-precolouring results, where no discernible improvement is observed.

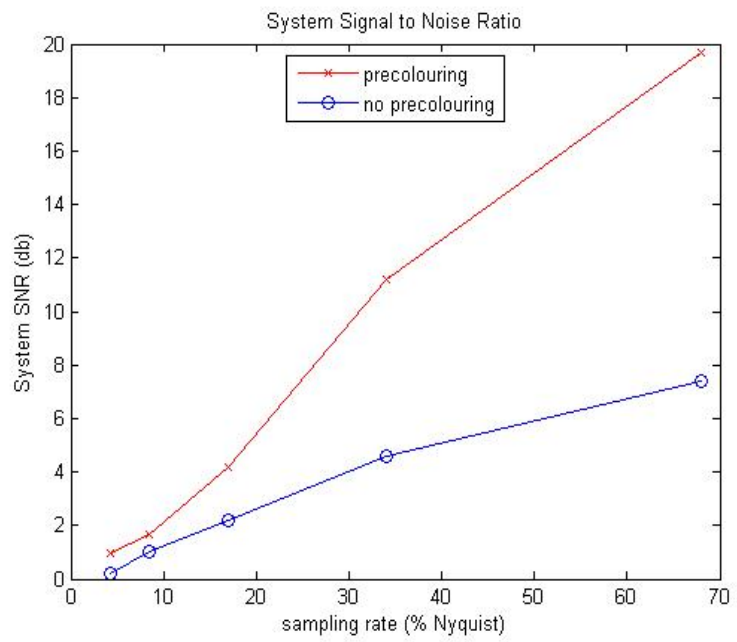

Figure 4. The effect of precolouring on SNR

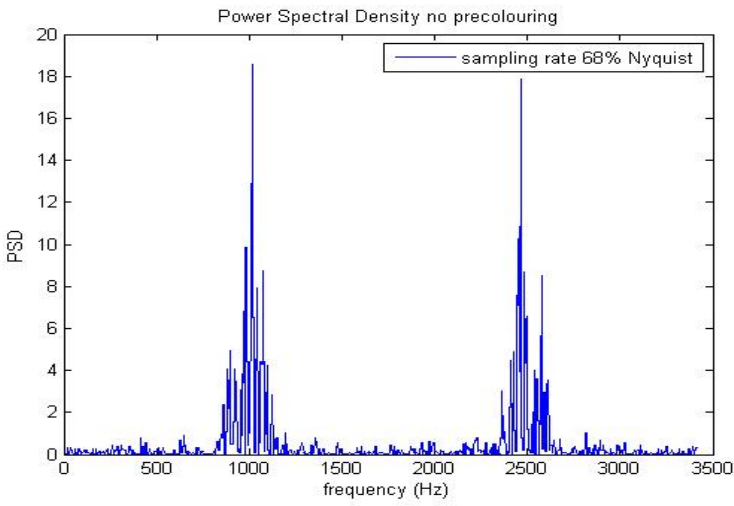

(a)

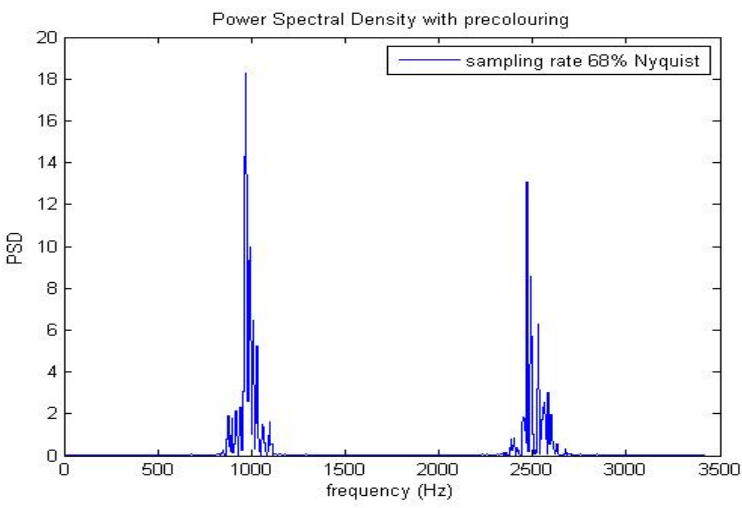

(b)

Figure 5. The recovered normalised PSD (W/Hz) at a sampling frequency of the $68 \%$ Nyquist with (a) no precolouring and (b) AR filter precolouring

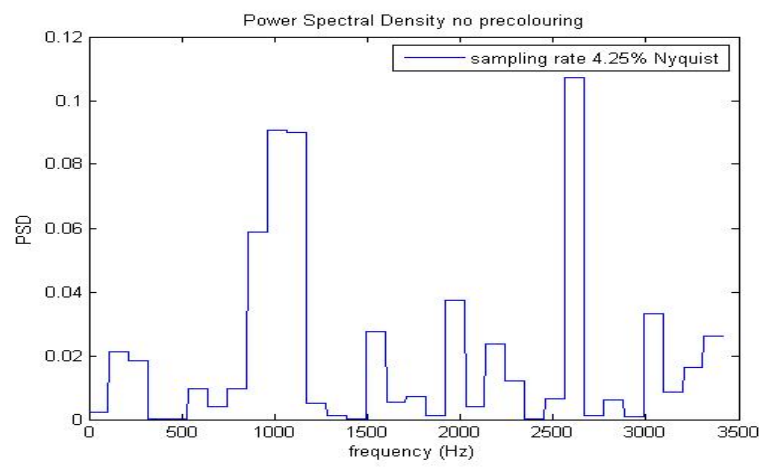

(a)

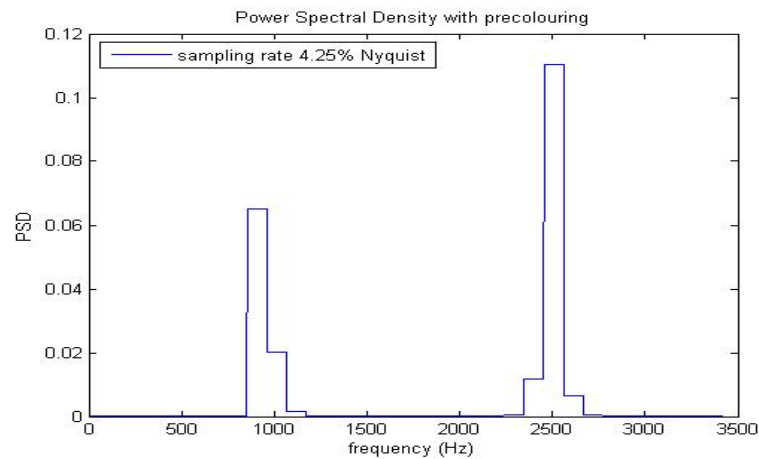

(b)

Figure 6. Recovered normalised PSD (W/Hz) at sampling rates 4.25\% Nyquist with (a) no precolouring, (b) AR filter precolouring

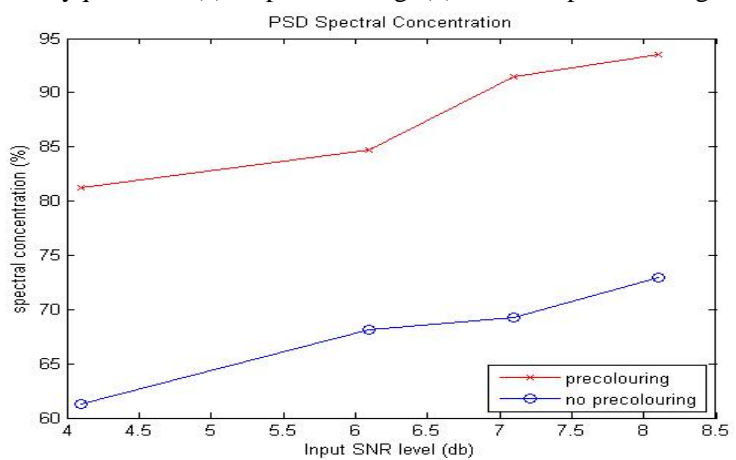

(a)

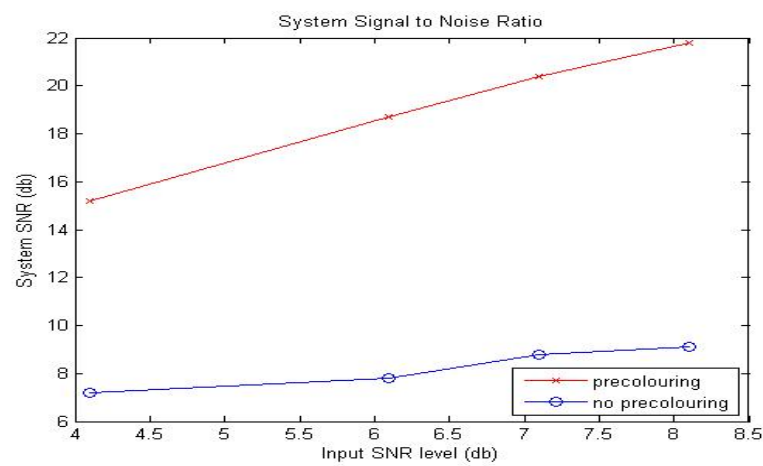

(b)

Figure 7. PSD Spectral Concentration for the (a) SNR (b) behaviour for variable levels of AWG noise to the input signal 


\section{CONCLUSION}

This paper has presented a novel compressive spectrum estimation model for sparse multiband signals which is particularly applicable in cognitive radio (CR) applications. Using a combination of a random demodulator (RD) architecture and autoregressive precolouring filter, input signals are sub-Nyquist sampled and their sharpened power spectral density (PSD) estimated and recovered by a $l_{1}$-norm minimization algorithm and inverse filter. Experimental results for quadrature-phased-shift-keying (QPSK) modulated multiband signals have conclusively shown that at different sub-sampling rates and input noise levels, the proposed compressive sensing (CS) spectrum estimation structure consistently and robustly reduced PSD spectral leakage, while concomitantly providing superior signal-to-noise ratio performance. Future development of this model will focus upon extending the CS architecture to handle alternative and higher-order modulation signal types, especially PSK and QAM. It will also seek to analyze the sensitivity of the proposed compressive spectrum estimation model to the choice of AR filter order $p$ and the comparative behaviour and performance of alternative CS techniques including the Compressive Multiplexor and the Modulated Wideband Converter.

\section{REFERENCES}

[1] S. Haykin, "Cognitive radio: Brain-empowered wireles communications”, IEEE Journal on Selected Areas in Communications, Vol.23, No.2, Feb. 2005.

[2] D. Cabric, S.M. Mishra, R.W. Brodersen, "Implementation issues in spectrum sensing for cognitive radios", $38^{\text {th }}$ Asilomar Conference on Signals, Systems and Computers, vol.1, pp.772-776, Nov. 2004

[3] Z.Tian, G. B. Giannakis, “Compressed sensing for wideband cognitive radios”, IEEE International Conference on Acoustics, Speech and Signal Processing, pp. IV-1357-IV-1360, Apr. 2007.

[4] E. Candès, E. Wakin, "An Introduction To Compressive Sampling”, IEEE Signal Processing Magazine, Vol.25, Issue 2, pp. 21-30, Mar. 2008.

[5] E. J. Candès, J. Romberg, T. Tao, "Robust uncertainty principles: Exact signal reconstruction from highly incomplete frequency information", IEEE Trans. Information Theory, vol. 52, no. 2, pp. 489-502, Feb. 2006.

[6] D. L. Donoho, “Compressed sensing”, IEEE Transactions on Information Theory, vol. 52, pp. 1289-1306, Apr. 2006.

[7] Federal Communications Commission, “ Spectrum Policy Task Force ,’ Rep. ET Docket no. 02-135, Nov. 2002.

[8] M. Vetterli, P. Marziliano, and T. Blu, "Sampling signals with finite rate of innovation,” IEEE Transactions on Signal Processing, vol. 50, no. 6 , pp. 1417-1428, Jun. 2002.

[9] S. Kirolos, J. Laska, M. Wakin, M. Duarte, D. Baron, T. Ragheb, Y. Massoud and R. Baraniuk, "Analog-to-Information Conversion via Random Demodulation”, IEEE Workshop on Design, Applications, Integration and Software, pp.71-71, Oct. 2006

[10] J. N. Laska, S. Kirolos, M. F. Duarte, T. S. Ragheb, R. G. Baraniuk and Y. Massoud, "Theory and implementation of an analog-to-information converter using random demodulation”, IEEE International Symposium on Circuits and Systems, pp. 1959-1962, May 2007.

[11] T. Ragheb, J. N. Laska, H. Nejati, S. Kirolos, R. G. Baraniuk, and Y. Massoud "A prototype hardware for random demodulation based compressive Analog-to-Digital Conversion”, 51st IEEE Midwest Symposium in Circuits and Systems, pp. 37-40, Aug. 2008.

[12] J. A. Tropp, J. N. Laska, M. F. Duarte, J. K. Romberg and R. G Baraniuk, "Beyond Nyquist: Efficient sampling of sparse bandlimited signals", IEEE Transactions On Information Theory, Vol. 56, No. 1, pp.520-544, Jan. 2010.

[13] Y. Eldar \& G. Kutyniok, "Compressed Sensing, Theory and Applications”, Cambridge, UK, Cambridge University Press, 2012.

[14] Z. Yu, S. Hoyos and B.M. Sadler, "Mixed - signal parallel compressed sensing and reception for cognitive radio", IEEE International Conference on Acoustics, Speech and Signal Processing, pp.3861-3864, Mar-Apr. 2008.

[15] Z. Yu, S. Hoyos, "Compressive sensing front-ends for cognitive radio", International Conference on Systems, Man and Cybernetics, pp. 18991904, Oct. 2009.

[16] Y. Lu, W. Guo, X. Wing and W. Wang, "Distributed streaming compressive spectrum sensing for wide-band cognitive radio networks", $73^{\text {rd }}$ IEEE Vehicular Technology Conference, pp. 1-5, May 2011.

[17] M. Nekovee, "A survey of cognitive radio access to TV white spaces", Hindawi Publishing Corporation, International Journal of Digital Media Broadcasting, Vol. 2010, Article ID 236568, Apr. 2010

[18] A. N. Mody, G.Chouinard, "IEEE 802.22 Wireless regional area networks”, IEEE 802.22-10/0073r03, Jun. 2010.

[19] 11 Magic, a collection of MATLAB routines for solving the convex optimization programs central to compressive sampling. [Online]. Available: http://www.l1-magic.org/, (Accessed 12-12-2012).

[20] J. Romberg, “ Sensing by random convolution”, $2^{\text {nd }}$ IEEE International Workshop on Computational Advances in Multi-Sensor Adaptive Processing, pp. 137-140, Dec. 2007

[21] R. Baraniuk, M. A. Davenport, M. F. Duarte and C. Hegde, "An Introduction to Compressive Sensing”. [Online]. Available: http://cnx.org/content/col11133/1.5, (Accessed 12-12-2012).

[22] S. Haykin, “Adaptive Radar Signal Processing”, Hoboken, New Jersey, USA, John Wiley \& Sons, 2007.

[23] D. Manolakis, V. Ingle, S. Kogon, "Statistical and Adaptive Signal Processing”, Artech House, Boston, USA, 2005.

[24] S.L. Marple, “Digital Spectral Analysis with Applications”, Englewood Cliffs, New Jersey, USA, Prentice-Hall, 1987. 